\title{
Evaluation of turkey arthritis syndrome by traditional and advanced
} diagnostic techniques

Azza S. El-Demerdash ${ }^{1}$, Rehab E. Mowafy ${ }^{1 *}$, Sahar N.Mohamady ${ }^{1}$, Sanaa M.Salem1, Samah F. Ali ${ }^{2}$, ElToukhy. E.I ${ }^{2}$

${ }^{1}$ Agriculture Research center (ARC), Animal Health Research Institute (AHRI), Zagazig, Egypt

${ }^{2}$ Agriculture Research center (ARC), Animal Health Research Institute (AHRI), Dokki, Egypt

\section{ARTICLE HISTORY}

Received: 07.09.2020

Revised: 11.10 .2020

Accepted: 02.11.2020

Address correspondence to Rehab E.Mowafy; Tel: +201065033865; E-mail: mowafyrehab@yahoo.com

\section{ABSTRACT}

\begin{abstract}
Objective: To detect possible causes of arthritis in turkey flocks with emphasis on molecular detection of bacterial pathogens and estimation of their pathology pattern. Design: Descriptive study

Procedure: A total of 100 arthritic legs from 125 French turkey birds were collected from different farms in Sharkia Governorate. Specimens from joint capsule, tendons, and synovial fluid of leg joints were subjected to bacteriological and direct PCR examinations. Moreover, skin, cartilage of articular surface and epiphysis were undergone pathological examination. Blood samples were collected before scarification for both hematological and biochemical examinations.

Results: Forty percent (40\%) bacterial incidence rate including Staphylococcus spp., E. coli, Salmonella spp., Klebsiella spp., and Pseudomonas aeruginosa were recorded. Molecular detection of these pathogens revealed rapid and more accurate results of $46 \%$. A high prevalence of multidrug resistance was detected, but all isolates were susceptible to amikacin and florfenicol. Genotyping of isolates revealed the presence of strong virulence markers. Postmortem examinations revealed joints swelling, hyperkeratosis, erosions, and/or ulcerations. Further investigations on positive infected samples revealed a significant decrease in RBCs, Hb, PCV \%, lymphocytes, total protein, and albumin $(P<0.05)$. However, there was a significant increase in total leukocytic count and heterophile, CRP, ALT, AST, uric acid, creatinine, and total globulin $(P<0.05)$. Epidermal leukocytic cells infiltrations, tendonitis, synovium fibrosis, erosions, and/or ulcerations of articular cartilage were recorded.

Conclusion: Turkey arthritis is a serious problem facing turkey flocks in Egypt. Multiplex real-time PCR assay offered an effective alternative to traditional typing methods for the identification of the etiological agents involved in the infectious arthritis in turkey.
\end{abstract}

Keywords: Turkey, Arthritis, Bacterial pathogens, multiplex real-time PCR, Histopathology
ABBREVIATIONS: PCR: Polymerase chain reaction, CNS: Coagulase -negative staphylococci, RBCs: Red blood cells, $\mathrm{Hb}$ : Hemoglobin, PCV: Packed cell volume, CRP: C-reactive protein, AST: Serum aspartate aminotransferase, ALT: Alanine aminotransferase

\section{INTRODUCTION}

Arthritis is a worldwide welfare issue in poultry production especially turkeys, caused by many bacterial pathogens [1].The most common form of infection involves tenosynovitis (inflammation of tendon sheaths) and arthritis of the hock and stifle joints [2]. The affected joints, usually the hocks, are hot, swollen and painful and affected birds are usually depressed, lameness and reluctant to walk-in tenosynovitis, synovial membranes of tendon sheaths become thickened and edematous, with fibrinous exudate within and around the tendon sheaths leading to reduction of productivity, besides representing a sanitary problem [3]. As most bacterial pathogens could play a role in the incidence of respiratory disease in birds. The respiratory tract infections are of eminent importance in the manufacture of turkeys because of high mortality in poorly managed cases which lead to large economic losses [3].

The most important locomotor affection is septic arthritis which means a microbial infection of one or more joints and characterized pathologically by abscess foci formation either broken with deep ulcer and thickened skin or gangrenous dermatitis. Abscesses contained solidified cheesy-like material with ulcerative epithelial damage, marked hyperkeratosis, and extensive dermal suppuration with the presence of granular basophilic structures microscopically; probable to be bacterial colonies. Extensive fibrous connective tissue proliferation and dermal angiogenesis were also detected [4]. Multifocal degenerative changes in the articular surfaces (fibrosis, metaplasia of cartilage and bone), enlarged joints, thick friable 
tissue around the joint and severe articular erosions, a severe proliferation of fibrous connective tissue in /around articular surfaces, severe keratopathy with thick dull yellow exudate around the joints associated with mixed bacterial infection [5].

Also, measurement hematological parameters and CRP play a significant role in the diagnosis of this disease. CRP is synthesized in the liver and its physiologic role is to bind to phosphocholine expressed on the surface of dead or dying (apoptosis) cells to activate the complement / immune system, which enhances phagocytosis by macrophages [6]. Levels of CRP begin to rise within 2 hours. The rapid action of CRP makes it a participant in the acute or first phase of the inflammatory process, which is why it is often called an "acute-phase protein" $[7,8]$.

Therefore, the purpose of our study was to validate the recent and traditional techniques in investigation and diagnosis of the microbial communities as well as the hematological, biochemical, and histopathological changes accompanied by turkey arthritis syndrome that unfortunately hasn't received great attention and discussed in very little papers. Additionally, it was crucial to find the drug of choice in light of the random use of antibiotics which resulted in severe and multiple resistant strains to overcome this economic problem.

\section{MATERIALS AND METHODS}

\subsection{Field samples}

One hundred and twenty-five French turkey birds (Hybrid Grade Maker, Hybrid Optima, Hybrid Converter and Hybrid XL breeds) of ages varied from 60 to 185 days from both sex suffering from lameness with swollen joints were collected from different farms in Sharkia governorate.

Two blood samples were collected from the wing vein of each bird: the first sample was collected on di-potassium ethylene diamine tetraacetate (EDTA) as an anticoagulant to covering out hematological examination and the second one was collected in a clean, dry centrifuge tube without anticoagulant, left to clot at room temperature then centrifuged at 3000 rpm for 15 minutes. The sera were collected for biochemical studies.

Tissue samples were collected from the arthritic joints of both freshly dead and sacrificed birds under complete septic conditions after the disinfection of the joint externally with alcohol for microbiological examination by traditional methods, direct PCR examination for bacterial pathogens. Finally collected joints tissues were fixed in $10 \%$ formalin for histopathological examination.

\subsection{Bacterial isolation and identification}

Samples were enriched in nutrient broth at $37^{\circ} \mathrm{C}$ for $24 \mathrm{~h}$ then inoculated into Tryptic Soya Broth and Rappaport Vassiliadis (for Salmonella enrichment) then subjected to conventional methods for isolation and identification according to methodology recommended by Quinn et al.[9].

\subsection{Antibacterial susceptibility testing}

Antibacterial sensitivity tests were carried on the obtained isolates using the disk diffusion method on Mueller-Hinton Agar (OXOID), according to the procedure recommended by the Clinical and Laboratory Standards Institute (CLSI [10]). All isolates were tested for various routine antimicrobial drugs (OXOID), the tested antibiotics and their concentrations on $\mu \mathrm{g} /$ disk were as following: streptomycin (S; 10), amikacin (AK; 30), novobiocin (NV; 30), amoxicillin-clavulanic acid (AMC; 30), ampicillin (AMP; $10)$, florfenicol (FLO; 30), neomycin ( $N ; 30)$, lincomycin ( $; ; 2)$, doxycycline (DO;30) and colistin (CT;10). The inhibition zones, in millimeters, were measured in duplicate and scored as sensitive, intermediate, and resistant categories following the critical breakpoints recommended by the Clinical and Laboratory Standards Institute.

\subsection{Molecular characterization}

\subsubsection{Genomic extraction}

The DNA was extracted from samples using the QIAamp DNA Mini kit (Qiagen, Germany, $\mathrm{GmbH}$ ) according to the manufacturer's recommendations.

\subsubsection{Primer specificity}

Oligonucleotide primer sets that specifically amplify the target sequences of Virtual primers specificity was checked in a BLAST search available through the National Centre for Biotechnology Information (NCBI), website www.ncbi.nlm.nih.gov, before they were synthesized at Bio Basic, Canada INC. Further, six reference strains of $S$. aureus, coagulase-negative staphylococci, E. coli, Salmonella spp., Klebsiella pneumoniae, Pseudomonas aeruginosa were used for testing the specificity of primers as well as positive controls.

\subsection{3. $P C R$ amplification by multiplex real-time PCR direct from samples}

The multiplex real-time PCR assay was simultaneously carried out in duplicate for the detection of 6 tested bacterial pathogens. The optimal PCR amplification reaction mixture contained 12.5 master mix (Sensifast Prob, No Rox, Bio-86050), $1 \mu \mathrm{L}$ of 100 pmole of each primer (Sigma, USA), $1 \mu \mathrm{L}$ of 50 pmole of each primer, $6.5 \mu$ Lof template DNA. PCR amplification was performed in one step thermocycler (Germany) with the following cycling conditions at $95{ }^{\circ} \mathrm{C}$ for $5 \mathrm{~min}, 95^{\circ} \mathrm{C}$ for $10 \mathrm{sec}$, annealing $60{ }^{\circ} \mathrm{C}$ for $50 \mathrm{sec}$, and repeated for 40 cycles. Positive controls (reference strains) and negative controls (containing no DNA) were included in each assay run.

\subsection{4. $P C R$ amplification for some bacterial virulence genes by conventional $P C R$}


The multidrug -resistant bacterial isolates were screened for the presence of the major virulence factors with adjusting the final volume of the reaction mixture to $25 \mu \mathrm{L}$ consisting of 12.5 $\mu \mathrm{L}$ of DreamTaq TM Green Master Mix (2X) (Fermentas, USA), 1 $\mu \mathrm{L}$ of 100 pmole of each primer (Sigma, USA), $5 \mu$ Lof template DNA and water nuclease-free up to $25 \mu \mathrm{L}$. Target genes, primers sequences, amplified product size and cycling conditions were illustrated in Tables 1-3.

The products of PCR were separated by electrophoresis on $1.5 \%$ agarose gel (Applichem, Germany, GmbH) in TBE (1x) buffer. For gel analysis, $15 \mu$ l of the products were loaded in each gel slot. The gel was photographed by a gel documentation system (Alpha Innotech, Biometra) and the data was analyzed through computer software.

\subsection{Hematological studies}

The RBCs count, $\mathrm{Hb}$ concentration ( $\mathrm{gm} / \mathrm{dl}$ ), PCV (\%), and the total and differential leukocytic counts were measured according to Thrall et al. [11]

\subsection{Biochemical studies}

The CRP was determined by ELISA Kits according to Banerjee et al. [12]. AST and ALT activities were measured according to Murray [13] . Serum uric acid was determined according to Young [14], serum creatinine level according to Burtis \& Ashwood [15], total serum protein was measured according to Doumas et al.[16], albumin was determined according to Drupt [17], globulin was calculated as the difference between total protein and albumin.

\subsection{Gross lesions and histopathology}

Specimens in the form of several cut sections from the affected joints (stifle joints, hock joints and metatarsal joints) including different parts as; skin, tendons, cartilages, and epiphysis were collected from sacrificed turkey birds suffering from arthritic joints. Specimens were fixed in $10 \%$ neutral buffered formalin for histopathological examination. Paraffin tissue sections of 5-7 micron thick were prepared and stained with hematoxylin and eosin then examined microscopically [18].

\section{8. statistical analysis}

Data analysis was performed by SPSS version 22 for windows. One-way ANOVA was used to assess hematological and biochemical parameters. Also, the proportion difference between PCR and re-isolation results was done by using medcalc statistical software. Chi-Square Test was used to evaluate if the difference between traditional and molecular assays were statistically significant or not. A P $<0.05$ value was considered statistically significant.

\section{RESULTS}

\subsection{Postmortem lesions}

Various pictures and variable degrees of joints swelling and toes curling were observed in the examined birds. The gastrocnemius tendon sheath along the hock join, the proximal tarsometatarsal joints were the most affected leg parts. Our results revealed skin with hyperkeratosis (thickened, cracked and tough skin) in most cases with ulceration few cases revealed suppuration. Open joints and tendon sheaths with viscid grey to yellow turbid exudate in positive bacterial infection cases were observed, Suspected synovium fibrosis (stretched, decreased size and depressed when compared to normal areas are could be observed in some chronic non-treated cases. Gross lesions associated with different isolated microbes were demonstrated in detail in lesions score (Table. 4) and some of these forms of injuries were demonstrated in Figure 1.

Table 4. Lesion score of different articular parts in turkey suffering septic arthritis.

\begin{tabular}{|c|c|c|c|}
\hline $\begin{array}{l}\text { Affected } \\
\text { parts }\end{array}$ & Lesions & Types of infection & $\begin{array}{l}\text { Lesion } \\
\text { Severity }\end{array}$ \\
\hline \multirow[t]{4}{*}{ Skin } & $\begin{array}{l}\text { Dermal } \\
\text { hyperkeratosis }\end{array}$ & $\begin{array}{l}\text { All mixed infection with Staph } \\
\text { spp. }\end{array}$ & +++ \\
\hline & $\begin{array}{l}\text { Subdermal } \\
\text { edema }\end{array}$ & $\begin{array}{l}\text { All mixed infection included } \\
\text { Staph spp. }\end{array}$ & ++ \\
\hline & $\begin{array}{l}\text { Heterophile } \\
\text { cells Infiltration }\end{array}$ & All bacterial infection & +++ \\
\hline & $\begin{array}{l}\text { Lymphocytic } \\
\text { cells infiltration }\end{array}$ & All pathogens & +++ \\
\hline \multirow{3}{*}{$\begin{array}{l}\text { Tendons } \\
\text { and } \\
\text { Synovium }\end{array}$} & Tendonitis & All mixed infection & ++ \\
\hline & Fibrosis & All bacterial infection & ++ \\
\hline & $\begin{array}{l}\text { Heterophile } \\
\text { cells Infiltration }\end{array}$ & All single and mixed infection & +++ \\
\hline \multirow[t]{2}{*}{ Cartilage } & $\begin{array}{l}\text { Necrosis of } \\
\text { chondrocytes }\end{array}$ & All bacterial infection & ++ \\
\hline & $\begin{array}{l}\text { Erosions and } \\
\text { ulcers }\end{array}$ & $\begin{array}{l}\text { All mixed infection included } \\
\text { Staph spp. }\end{array}$ & ++ \\
\hline \multirow[t]{3}{*}{ Bone } & $\begin{array}{c}\text { Decalcification } \\
\text { Epiphyseal }\end{array}$ & All bacterial cases & + \\
\hline & cyst & & \\
\hline & $\begin{array}{l}\text { Heterophile } \\
\text { cells } \\
\text { Infiltration }\end{array}$ & All bacterial infection & +++ \\
\hline Severe +++ & Moder & Mild + & \\
\hline
\end{tabular}

\subsection{The recovery rate of isolation and identification}

Bacteriological examination revealed that $40 \%$ of collected samples were positive. They were identified as Staphylococcus aureus (27\%), followed by and coagulase-negative Staphylococcus (CNS) and Salmonella spp with a percent of $15 \%$ for each, other bacterial infections were also recorded, but with lower percentages, as shown in Table 5.

Regarding molecular examination, $46 \%$ of samples were positive for arthritis representing significantly higher percentages than those detected by traditional methods. 
Overall, according to PCR results, 13 samples only represent a single infection and the rest represents the mixed ones (Figure 2).

Table 1. Primers and probes sequence for real-time PCR amplification

\begin{tabular}{|c|c|c|}
\hline Microorganism & Oligo name & Sequence $5^{\prime \prime}-3^{\prime \prime}$ \\
\hline \multirow[t]{3}{*}{ E. coli } & E. coli.F & ATCGTGACCACCTTGATT \\
\hline & E. coli.R & TACCAGAAGATCGACATC \\
\hline & E. coli.Pro. & HEX-CATTATGTTTGCCGGTATCCGTTT- BHQ2 \\
\hline \multirow[t]{3}{*}{ S. aureus } & S.aureus.F & ATCGTGACCACCTTGATT \\
\hline & S.aureus. $R$ & TACCAGAAGATCGACATC \\
\hline & S.aureus.Pro & HEX-CATTATGTTTGCCGGTATCCGTTT-BHQ1 \\
\hline \multirow[t]{3}{*}{ Pseudomonas } & Pseudomonas.F & CCTGACCATCCGTCGCCACAAC CGCAGCAGGATGCCGACGCC \\
\hline & Pseudomonas. $R$ & FAM-CCGTGGTGGTAGACCTGTTCCCAGACC-BHQ1 \\
\hline & Pseudomonas.Pro & \\
\hline \multirow[t]{3}{*}{ CN.staph } & CN.staph.F & GGGTGACTAACAGTGGA \\
\hline & CN.staph.R & GCGGATCCATCTAAGTGA \\
\hline & CN.staph.Pro. & FAM-GGATAATATATTGAACCGCA-BHQ1 \\
\hline \multirow[t]{3}{*}{ Salmonella } & Salmonella.F & GGAGTATGGTTGCAAAGCTGA \\
\hline & Salmonella.R & GGTGAGGTTTCCCGTGTTG \\
\hline & Salmonella.Pro & Cy5-AAGGAATTGACGGAAGGGCA-BHQ1 \\
\hline \multirow[t]{3}{*}{ Klebsiella } & Klebsiella.F & TCCGGGATGCGTGACGTTGC \\
\hline & Klebsiella.R & TGCTGCGCGAATGAAGACGA \\
\hline & Klebsiella.Pro & FAM-TCATGGAGAATCGCTGGGGGAAAGC-BHQ1 \\
\hline
\end{tabular}

Table 2. Primers sequence of the virulence markers of each pathogen

\begin{tabular}{|c|c|c|c|c|}
\hline Microorganism & Target gene & Primers sequences & $\begin{array}{l}\text { Length of amplified } \\
\text { product (bp) }\end{array}$ & Reference \\
\hline \multirow[t]{4}{*}{ Salmonella } & mgtC & TGA CTA TCA ATG CTC CAG TGA AT & 677 & Huehn et al.[46] \\
\hline & & ATT TAC TGG CCG CTA TGC TGT TG & & \\
\hline & sopB & TCA GAA GRC GTC TAA CCA CTC & 517 & \\
\hline & & TAC CGT CCT CAT GCA CAC TC & & \\
\hline \multirow[t]{4}{*}{ Staphycocci } & spa & TCA ACA AAG AAC AAC AAA ATG C & 226 & Wada et al. [47] \\
\hline & & GCT TTC GGT GCT TGA GAT TC & & \\
\hline & $i c a A$ & CCT AAC TAA CGA AAG GTA G & 1315 & Ciftci et al. [48] \\
\hline & & AAG ATA TAG CGA TAA GTG C & & \\
\hline \multirow[t]{4}{*}{ E. coli } & ompA & AGCTATCGCGATTGCAGTG & 919 & Ewers et al. [49] \\
\hline & & GGTGTTGCCAGTAACCGG & & \\
\hline & neuc & GGTGGTACATTCCGGGATGTC & 670 & \\
\hline & & AGGTGAAAAGCCTGGTAGTGT & & \\
\hline \multirow[t]{4}{*}{ P. aeruginosa } & aprA & GTCGACCAGGCGGCGGAGCAGATA & 993 & Sabharwal et al. [33] \\
\hline & & GCCGAGGCCGCCGTAGAGGATGTC & & \\
\hline & $\operatorname{tox} A$ & GGAGCGCAACTATCCCACT & 150 & \\
\hline & & GGTAGCCGACGAACACATA & & \\
\hline \multirow[t]{4}{*}{ Klebsiella spp. } & $\operatorname{mag} A$ & GGTGCTCTTTACATCATTGC & 1283 & Compain et al. [50] \\
\hline & & GCAATGGCCATTTGGGTTAG & & \\
\hline & $r m p A$ & CATAAGAGTATTGGTTGACAG & 461 & \\
\hline & & CTTGCATGAGCCATCTTTCA & & \\
\hline
\end{tabular}

\subsection{Antibiotic susceptibility results}

The tested pathogens showed different percentages of resistance and susceptibility to different antibiotics. All isolates revealed absolute resistance to amoxicillin-clavulanic acid and novobiocin. However, the isolates showed the ultimate susceptibility to amikacin and florfenicol, (Table 6).
Moreover, the multidrug -resistance phenomenon was observed through almost isolates including $23 \%$ of Staphylococci species (10/43), 40\% E. coli (4/10), 26\% Salmonella (4/15), 66.6\% Klebsiella spp. (2/3) and $50 \%$. aeruginosa (1/2).

\subsection{Virulence determinants of the obtained multidrug-resistant isolates}


The results indicated a clear abundance of these virulence genes, which were detected in 18 of 21 analyzed isolates (85.71\%). Regarding Salmonella isolates, $50 \%$ of isolates were harbored $\mathrm{mgtC}$ and the rest exhibited another tested gene $(s o p B)$. Among tested staphylococci species, all tested isolates harbored icaA and all examined $S$. aureus carried spa gene. Analyzing the PCR profiles of $4 E$. coli isolates revealed that the variations in genotypes to ompA and neuC genes where only one of them harbored both genes and the rest carried ompA gene alone. Furthermore, aprA and tox $A$ genes were simultaneously present in all tested $P$. aeruginosa isolates. Among the genes coded virulence profile of tested Klebsiella isolate, magA was only detectable and yielded a 1,283 bp specific sequence (Figure $\mathbf{3}$ ).

Table 3: PCR amplification and cycling protocol of virulence genes of bacterial pathogens

\begin{tabular}{|c|c|c|c|c|c|c|c|c|c|c|}
\hline \multirow{2}{*}{ Thermal profile } & \multicolumn{2}{|c|}{ Salmonella } & \multicolumn{2}{|c|}{ Staphycocci } & \multicolumn{2}{|l|}{ E.coli } & \multicolumn{2}{|c|}{$P$. aeruginosa } & \multicolumn{2}{|c|}{ Klebsiella spp. } \\
\hline & mgtC & sopB & Spa & $i c a A$ & $o m p A$ & neuc & aprA & tox $A$ & $\operatorname{magA}$ & $r m p A$ \\
\hline \multirow{2}{*}{ Initial denaturation } & $94 \div 0 \mathrm{C}$ & $94 \circ \mathrm{C}$ & $94^{\circ} \mathrm{C}$ & $94^{\circ} \mathrm{C}$ & $94^{\circ} \mathrm{C}$ & $94^{\circ} \mathrm{C}$ & $95^{\circ} \mathrm{C}$ & $95^{\circ} \mathrm{C}$ & $95^{\circ} \mathrm{C}$ & $95^{\circ} \mathrm{C}$ \\
\hline & $5 \mathrm{~min}$ & $5 \mathrm{~min}$ & $5 \mathrm{~min}$ & $5 \mathrm{~min}$ & $5 \mathrm{~min}$ & $5 \mathrm{~min}$ & $2 \min$ & $2 \min$ & $15 \min$ & $15 \mathrm{~min}$ \\
\hline \multirow{2}{*}{ Denaturation } & $94 \circ \mathrm{C}$ & $94 \stackrel{\circ}{ } \mathrm{C}$ & $94^{\circ} \mathrm{C}$ & $94^{\circ} \mathrm{C}$ & $94^{\circ} \mathrm{C}$ & $94^{\circ} \mathrm{C}$ & $95^{\circ} \mathrm{C}$ & $95^{\circ} \mathrm{C}$ & $94^{\circ} \mathrm{C}$ & $94^{\circ} \mathrm{C}$ \\
\hline & $30 \mathrm{sec}$ & $30 \mathrm{sec}$ & $30 \mathrm{sec}$. & $30 \mathrm{sec}$ & $30 \mathrm{sec}$ & $30 \mathrm{sec}$ & $40 \mathrm{sec}$ & $40 \mathrm{sec}$ & $30 \mathrm{sec}$ & $30 \mathrm{sec}$ \\
\hline \multirow{2}{*}{ Annealing } & $58 \circ \mathrm{C}$ & $58 ㅇ$ & $55^{\circ} \mathrm{C}$ & $49^{\circ} \mathrm{C}$ & $55^{\circ} \mathrm{C}$ & $60^{\circ} \mathrm{C}$ & $50^{\circ} \mathrm{C}$ & $65^{\circ} \mathrm{C}$ & $55^{\circ} \mathrm{C}$ & $55^{\circ} \mathrm{C}$ \\
\hline & $45 \mathrm{sec}$ & $45 \mathrm{sec}$ & $30 \mathrm{sec}$ & $1 \mathrm{~min}$ & $40 \mathrm{sec}$ & $40 \mathrm{sec}$ & $1 \mathrm{~min}$ & $1 \mathrm{~min}$ & $30 \mathrm{sec}$ & $30 \mathrm{sec}$ \\
\hline \multirow{2}{*}{ Extension } & $72{ }^{\circ} \mathrm{C}$ & $72 \stackrel{\circ}{ } \mathrm{C}$ & $72^{\circ} \mathrm{C}$ & $72^{\circ} \mathrm{C}$ & $72^{\circ} \mathrm{C}$ & $72^{\circ} \mathrm{C}$ & $72^{\circ} \mathrm{C}$ & $72^{\circ} \mathrm{C}$ & $72^{\circ} \mathrm{C}$ & $72^{\circ} \mathrm{C}$ \\
\hline & $45 \mathrm{sec}$ & $45 \mathrm{sec}$ & $30 \mathrm{sec}$ & $1 \mathrm{~min}$ & $45 \mathrm{sec}$ & $45 \mathrm{sec}$ & $2 \min$ & $2 \min$ & $30 \mathrm{sec}$ & $30 \mathrm{sec}$ \\
\hline \multirow{2}{*}{ Final extension } & $72^{\circ} \mathrm{C}$ & $72 \circ \mathrm{C}$ & $72^{\circ} \mathrm{C}$ & $72^{\circ} \mathrm{C}$ & $72^{\circ} \mathrm{C}$ & $72^{\circ} \mathrm{C}$ & $72^{\circ} \mathrm{C}$ & $72^{\circ} \mathrm{C}$ & $72^{\circ} \mathrm{C}$ & $72^{\circ} \mathrm{C}$ \\
\hline & $10 \mathrm{~min}$ & $10 \min$ & $7 \mathrm{~min}$ & $12 \min$ & $10 \mathrm{~min}$ & $10 \min$ & $10 \mathrm{~min}$ & $10 \min$ & $7 \mathrm{~min}$ & $7 \mathrm{~min}$ \\
\hline Amplification cycles & 35 & 35 & 35 & 35 & 35 & 35 & 30 & 30 & 35 & 35 \\
\hline
\end{tabular}

Table 6. Antibiograms of different bacterial pathogens

\begin{tabular}{|c|c|c|c|c|c|c|c|c|c|c|}
\hline \multirow[t]{2}{*}{ Microorganism } & \multicolumn{10}{|c|}{ Number of resistant isolates to each antimicrobial agent } \\
\hline & S & AK & NV & $\mathrm{AMC}$ & AMP & FLO & $\mathrm{N}$ & $\mathrm{L}$ & DO & $\mathrm{CT}$ \\
\hline S. aureus (27) & 10 & 0 & 27 & 27 & 11 & 0 & 10 & 11 & 9 & 12 \\
\hline CNS (15) & 5 & 0 & 15 & 15 & 5 & 0 & 4 & 5 & 5 & 5 \\
\hline E. coli (10) & 7 & 0 & 10 & 10 & 4 & 0 & 3 & 4 & 4 & 4 \\
\hline Salmonella (15) & 6 & 0 & 15 & 15 & 6 & 0 & 6 & 7 & 6 & 6 \\
\hline Klebsiella (3) & 2 & 0 & 3 & 3 & 1 & 0 & 2 & 2 & 1 & 2 \\
\hline$P$. aeruginosa (2) & 1 & 0 & 2 & 2 & 1 & 0 & 1 & 1 & 1 & 1 \\
\hline Total (72) & 31 & 0 & 72 & 72 & 28 & 0 & 26 & 30 & 26 & 30 \\
\hline \% Resistant* & 43.05 & 0 & 100 & 100 & 39.4 & 0 & 36.6 & 41.6 & 36.6 & 41.6 \\
\hline$\%$ Intermediate & 38.88 & 0 & 0 & 0 & 16.66 & 0 & 12.5 & 44.44 & 44.44 & 30.55 \\
\hline$\%$ Susceptible & 18.07 & 100 & 0 & 0 & 43.93 & 100 & 50.9 & 13.9 & 18.95 & 27.84 \\
\hline
\end{tabular}

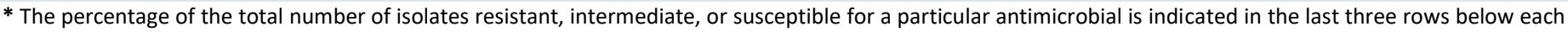
antimicrobial.

Table 5: Incidence rate of bacterial pathogens by traditional and molecular assays.

$\begin{array}{lll}\text { Bacterial pathogens } & \begin{array}{l}\text { Conventional culture } \\ \text { results }\end{array} & \text { PCR results } \\ \text { S. aureus } & 27 & 31 \\ \text { CNS } & 15 & 19 \\ \text { E. coli } & 10 & 12 \\ \text { Salmonella species } & 15 & 17 \\ \text { Klebsiella } & 3 & 4 \\ \text { P. aeruginosa } & 2 & 7 \\ \text { Total } & 72^{*} & 90^{*}\end{array}$

\footnotetext{
*: Indicates significant variations $(P$ value $<0.05)$
}

\subsection{Hematological parameters measurements}

In the present study, the hematological parameters showed a significant decrease in RBCs count, $\mathrm{Hb}$ concentration and PCV\% in the diseased birds. The leukogram of infected turkeys showed leukocytosis associated with heterophilia, monocytosis, and lymphopenia, non-significant change in basophils, and eosinophils (Table 7).

\subsection{The biochemical parameters}

The obtained results of the biochemical analysis in Table 8 revealed an increase in CRP, AST, and ALT activities. Also, a significant decrease in protein hypoproteinemia was detected. Hypoalbuminemia was observed as well as an increase of total 
globulin, serum uric acid, and creatinine in diseased turkeys compared to the control group.

Table 7. Hematological parameters (mean \pm SE) in turkey birds affected by infected group compared with control group $(\mathrm{N}=5)$

\begin{tabular}{|c|c|c|c|}
\hline \multicolumn{2}{|l|}{ Parameter } & Non-infected & Infected \\
\hline RBCs $\times 10^{6} /$ & $10^{6} / \mathrm{ul}$ & $2.2 \pm 0.11$ & $1.22 \pm 0.08 * * *$ \\
\hline $\mathrm{gm} / \mathrm{c}$ & $\mathrm{gm} / \mathrm{dl}$ & $9.69 \pm 0.34$ & $7.52 \pm 0.43^{* *}$ \\
\hline PCV & $\%$ & $30.08 \pm 1.05$ & $22.78 \pm 1.43^{* *}$ \\
\hline WBCs & $\times 10^{3} / \mathrm{UL}$ & $6.33 \pm 0.37$ & $7.8 \pm 0.30 *$ \\
\hline \multirow{2}{*}{$\begin{array}{l}\text { Heterophil } \\
\text { Lymphocytes }\end{array}$} & $\%$ & $31.69 \pm 0.84$ & $47.2 .4 \pm 2.44 * * *$ \\
\hline & $\%$ & $57.52 \pm 1.03$ & $37.8 \pm 2.33^{* * *}$ \\
\hline Monocytes & $\%$ & $4.46 \pm 0.18$ & $8.8 \pm 3.77 * *$ \\
\hline Basophil & $\%$ & $4.64 \pm 0.34$ & $4.2 \pm 0.32$ \\
\hline Eosinophil & $\%$ & $1.42 \pm 0.16$ & $2 \pm 0.55$ \\
\hline
\end{tabular}

Significant at $\mathrm{P}<0.001$

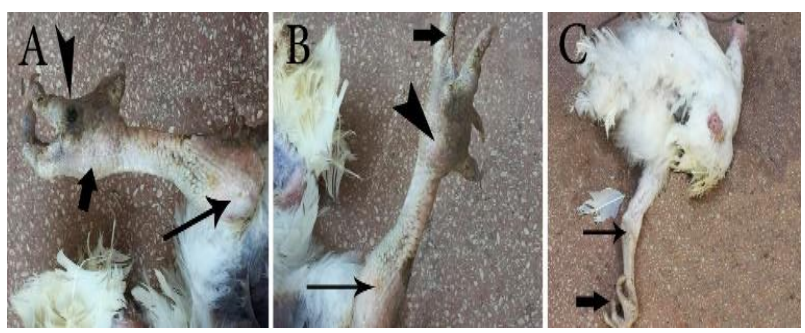

Figure 1: Lame legs of infected turkey (Hybrid Grade Maker, Hybrid Optima, and Hybrid XL breeds)suffering from septic arthritis showing; (A) Swollen hock joint (thin arrow) and intertarsal joints (thick arrow), necrotic area (head arrow), (B) Swollen hock joint (thin arrow) and intertarsal joints (head arrow), swollen toes (thick arrow), (C): swollen hock joint (thin arrow) and intertarsal joints with curved toes (thick arrow).

\subsection{Microscopical results}

Lame legs in infected arthritic turkeys revealed several forms of tissues injurious. The most commonly observed lesion in the skin was hyperkeratosis with/without focal areas of dermal papillae expansion into the epidermis (Fig.4a). The articular cartilage exhibited variable degrees of chondrocytes necrosis, while the joint capsule of most common cases revealed mononuclear leukocytic cells infiltration of mainly lymphocytes (Fig.4b) .Partial fibrosis of the synovium with or without mononuclear cells infiltration also observed (Fig.4c) .Another lesion of the articular cartilage exhibited multifocal erosions and/ or ulcerations (Fig.4d), moreover the epiphyseal (articular) bone of the affected joint showed focal decalcification with/without leukocytic cells infiltration(Fig.5a).Some articular joints showed moderate to severe congestion of tendon blood vessels (Fig.5b). In heavily infected cases joint epiphysis of injured joints showed cyst formation in the distorted epiphysis center (Fig.5c). Previously mentioned microscopical lesions were demonstrated in Figures (4\&5).
Table 8: Some Biochemical parameters of liver and kidney function (mean \pm SE) in serum of turkeys birds affected by bacterial arthritis compared with control group $(\mathrm{N}=5)$

$\begin{array}{lll}\text { Parameter } & \text { Non-infected } & \text { Infected } \\ & & \\ \text { CRP }(\mathrm{ng} / \mathrm{ml}) & 5.6 \pm 1.21 & 24 \pm 5.34^{* *} \\ \text { ALT (U/L) } & 11.54 \pm 0.95 & 23.25 \pm 1.98^{* * *} \\ \text { AST (U/L) } & 40.2 \pm 2.85 & 94.5 \pm 7.84^{* * *} \\ \text { Uric acid (mg/dl) } & 5.18 \pm 0.27 & 7.18 \pm 0.68^{*} \\ \text { Creatinine (mg/dl) } & 0.73 \pm 0.07 & 1.62 \pm 0.21 * * \\ \text { T.Protein }(\mathrm{g} / \mathrm{dl}) & 6.36 \pm 0.25 & 4.5 \pm 0.12^{* *} \\ \text { Albumin }(\mathrm{g} / \mathrm{dl}) & 3.52 \pm 0.14 & 0.85 \pm 0.08^{* * *} \\ \text { Globulin }(\mathrm{g} / \mathrm{dl}) & 2.84 \pm 0.21 & 3.65 \pm 0.19^{* *} \\ \text { A/G ratio } & 1.24 \pm 0.07 & 0.23 \pm 0.13^{* *}\end{array}$

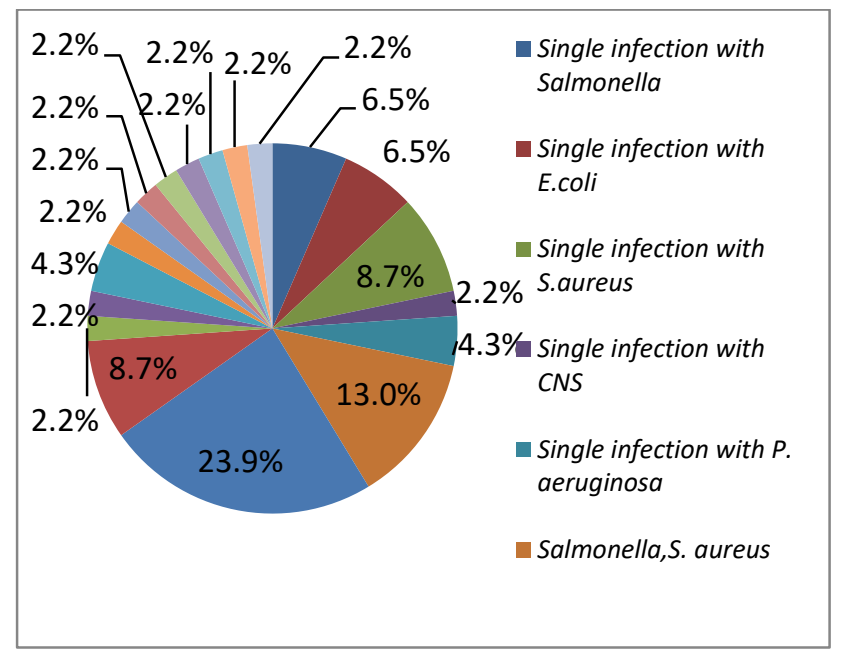

Figure 2: Molecular results of prevalence rate for single and mixed pathogens involved in arthritis by multiplex real-time PCR

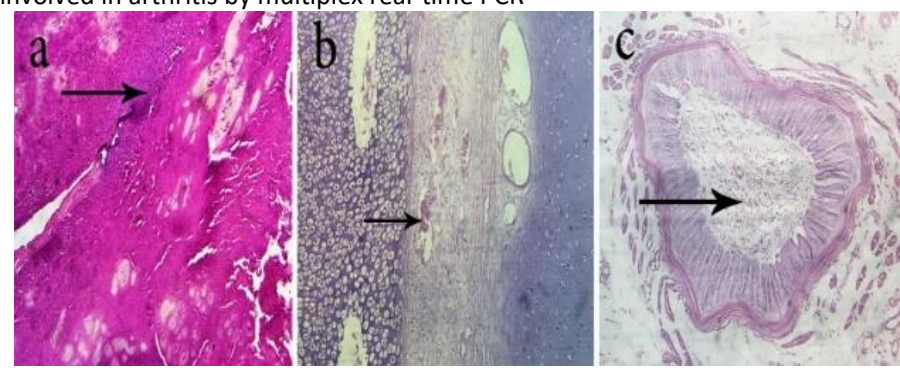

Figure 5. Sacrificed turkey joints suffering arthritis showing; (a) Photomicrograph of the epiphyseal (articular) bone of 8 months old Hybrid Optima turkey showing focal decalcification with leukocytic cells infiltration (arrow) (H\&E x100), (b) Photomicrograph of articular surface of 6 months aged Hybrid Grade Maker turkey showing congestion of tendon blood vessels (H\&E x100), (c) Photomicrograph of joint epiphysis of 9 months aged Hybrid converter turkey showing cyst formation in the distorted epiphysis center (H\&E x200). 


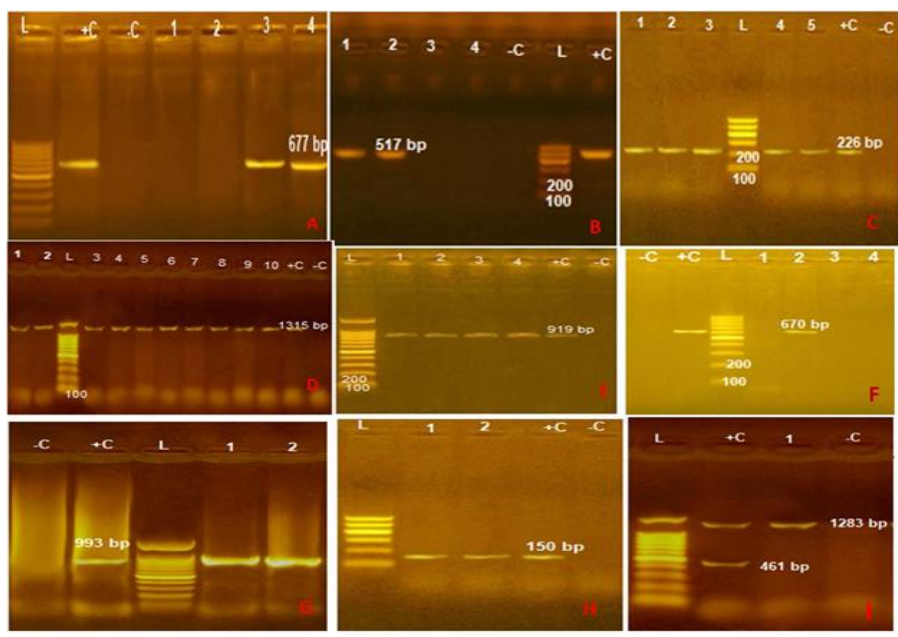

Figure 3. Agarose gel electrophoresis of DNA fragments generated of virulence markers of bacterial pathogens. Lane L: DNA molecular ladder (weight marker, 100 bp); Lanes -C: Negative control; Lanes +C: Positive control. (A, B) PCR amplification products with $\mathrm{mgtC}$ and sopB primers targeted 4 Salmonella isolates; (C) PCR amplification products with spa primer targeted $5 \mathrm{~S}$. aureus isolates, (D) PCR amplification products with icaA primer targeted 10 Staphylococci isolates ( $5 \mathrm{~S}$. aureus, $5 \mathrm{CNS}$ ) , (E, F) PCR amplification products with ompA and neuC targeted 4 E.coli isolates, (G, H) PCR amplification products with aprA and tox $A$ targeted $2 \mathrm{P}$. aeruginosa isolates, (I) Multiplex PCR amplification products with magA and rmpA generating the specific amplicons at 1283 and $461 \mathrm{bp}$, respectively targeted 1 Klebsiella isolate

\section{DISCUSSION}

Purulent arthritis, bacterial chondronecrosis with osteomyelitis were considered the most common causes of lameness in turkey breeds. In the present study, the isolation and identification results of bacterial agents using conventional methods depending on their phenotypic characteristics on the specific media for each microbe revealed that bacterial infection detected within the range of $40 \%$, and the most predominated organisms were Staphylococcus spp., Salmonella spp. in addition to E. coli. These data were fairly following Manohar et al. [19] and Kierończyk et al. [20] who demonstrated that multiple organisms can cause septic arthritis in poultry.

The PCR assays were optimized successfully and showed a significantly higher rat e of detection of arthritis bacterial pathogens singly and mixed in turkey than that was detected by the conventional culture methods and these findings were commonly reported by other investigators $[27,28]$.
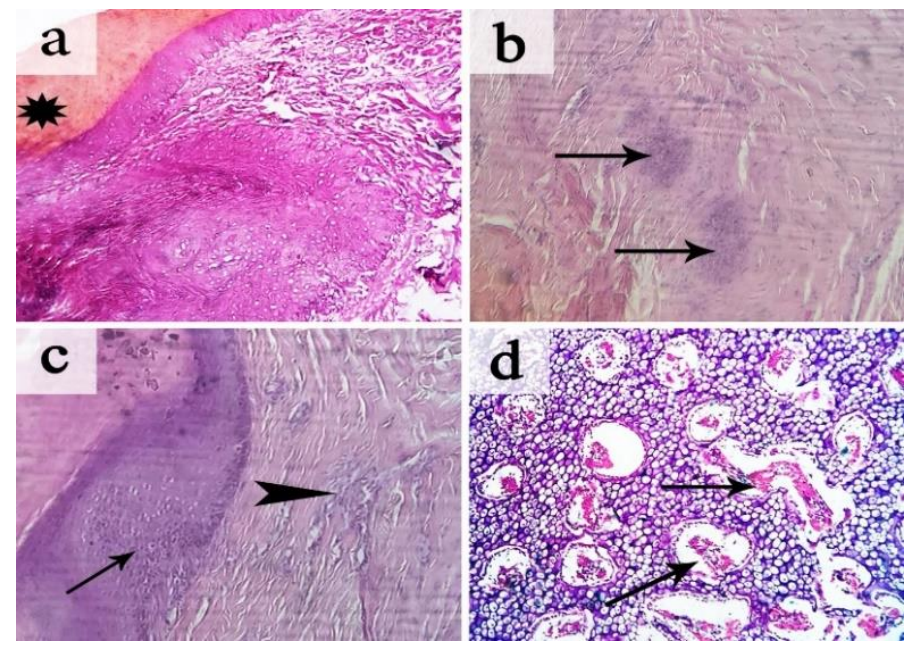

Figure 4. Sacrificed turkey joints suffering septic arthritis showing; (a) Hyperkeratosis (star) of 4 months aged Hybrid Grade Maker turkey with focal area of dermal papillae expansion into the epidermis (H\&Ex200), (b) Photomicrograph of the joint capsule 7 months aged Hybride Converter turkey showing mononuclear cells infiltration (arrows) (H\&Ex100), (c) Photomicrograph of the joint capsule 10 months aged Hybride Grade Maker turkey showing fibrosis of the synovium (head arrow) with mononuclear cells infiltration (arrow) (H\&E x100), (d) Photomicrograph of the articular cartilage of 13 months aged HybrideXL turkey showing multifocal erosions and ulcers (arrow) (H\&E x100).

The most frequent samples of mixed infections were $S$. aureus and CNS with a percentage $23.9 \%$ followed by $S$. aureus and Salmonella with a percentage $13 \%$. The obtained results were similar to those reported in South Africa, [3], Brazil [1], and the USA [23].

The presence of mixed infection in arthritic joint samples was proved in previous studies as Tawfik et al. [24] who mentioned that Salmonella spp. associated with arthritis in poultry is found either as the sole bacterium genus or as part of a cocktail of bacteria as $S$. aureus and E. coli. These data support the importance of joints as the site for isolation in screening livestock to identify emerging pathogens.

Concerning the antimicrobial susceptibility pattern of the obtained isolates, higher rates of sensitivity were observed to amikacin, florfenicol, and neomycin with percentages comparable to those found in many developing countries, especially Ghana [25] and Iran [26].

Also, relatively high level of antibiotic resistance among the examined isolates was detected especially to novobiocin, amoxicillin-clavulanic acid, streptomycin and colistin which mostly resulted from the long-term and widespread abuse of these antimicrobials in animal farms representing a significant disease burden in Egypt. Furthermore, 29.1\% (21/72) of tested isolates exhibited multidrug resistance, which was lower than that recorded in Japan and Nigeria by percentage $40.6 \%$ and $32.1 \%$, respectively. The consequence of this behavioral change over the years and countries is the gradual emergence of 
resistant bacterial strains refractory to multiple antimicrobial agents [27].

The large genome and its genetic complexity allow these pathogens to thrive in diverse ecological conditions. Multiple virulence factors impact their pathogenesis.

Regarding Salmonella, we examined the isolates for the sop $B$ and $m g t C$ genes, and the results revealed that each of them was detected in $50 \%$ of the isolates as compared with $100 \%$ of the isolates tested in India [28]. The $m g t C$ magnesium transport protein is a putative P-type ATPases which encodes a membrane protein that is indispensable for Salmonella survival in macrophages [29]. SopB gene implicated in the translocated effector protein of T3SS for SPI-5 translocated into the host cytosol, where it mediates inflammation and fluid secretion in the intestinal mucosa [30].

Molecular profile of examined staphylococci isolates ( $5 \mathrm{~S}$. aureus and 5 CNS) revealed that icaA detected in all of them and spa gene was detected in all examined $S$. aureus isolates (100\%) indicating high pathogenicity of these isolates represented in biofilm formation and spread in the host [31].

Concerning E.coli, ompA and neuC genes were detected with percentage $100 \%$ and $25 \%$, respectively indicating strong virulence biomarkers of the examined isolates. The perfect betabarrel structure of ompA is important for outer membrane stability and the presence of $\mathrm{K} 1$ capsule is encoded by neuC [32].

Furthermore, $100 \%$ prevalence of toxA and aprA genes in all analyzed $P$. aeruginosa strains promote the development of bacteria within the infected host and interfere with the host immune system [33].

Regarding K. pneumoniae isolate, magA gene was only detected which an important virulence gene in the host invasion, with a length of $1.2 \mathrm{~kb}$ and encoding an enzyme protein known as wzy, which acts as a polymerase in capsule synthesis. Therefore, the presence of magA in $K$. pneumoniae strain confers resistance to serum and phagocytosis [34]

The obtained findings were following Lucke et al. [35] and Youssef et al.[22], as they found a significant increase in total leukocytic counts, lymphocyte, and monocyte between infected and control groups especially in infected birds with $S$. aureus.

Biochemical analysis showed an increase in CRP in the infected group compared to the non-infected one. Furthermore, the increase in serum AST and ALT levels could be attributed to liver cells damage which may be produced by isolated bacteria [36] and [37] circulating in blood in both ascending and descending infection.

Also, the significant change in total protein, albumin may be due to bacterial toxins increase the capillary permeability and permitted the escape of plasma proteins into tissue resulting in hypoproteinemia [38]. Uric acid and creatinine in the present work could be increased due to liver and kidney damage which could be associated with a bacterial infection [22, 39].

The elevation of total globulin indicates an activation of the immune system which is due to infection or inflammatory diseases [40]. This increase might be attributed to the increased protein catabolism, febrile respiratory diseases, impaired cardiac function and decreased renal blood flow [22]. Zaki et al. [39] and Youssef et al. [22] mentioned that $S$. aureus is responsible for Bumble foot and septic arthritis in broilers and layers which cause a significant increase in AST, ALT, uric acid, creatinine, gamma, alpha globulin. Total protein and albumin levels were significantly decreased. These data were completely in accordance with our results.

Colored exudate in bacterial infection cases is typically detected by Echols [41] who proved that turbidity of synovial fluid can be another indicator of bacterial infection. Synovium fibrosis is a characteristic lesion of chronic cases as that reported by Davis et al. [41] and Eldin \& Ibrahim [43]. Some forms of grossly observed injuries are demonstrated in Figure1 are almost parallel to those obtained by Degernes et al. [5] and Hassan et al. [4]. Meanwhile, our microscopical results are in harmony with those recorded by some authors [49, 5, 4, 47, 48]. The obtained results of heterophilic and /or lymphocytic infiltration of the synovial membrane, synovial fold, and tendon collagen in most of the arthritic cases depends on the type of infection whatever bacterial or viral one consequently which supported by the results of previously mentioned authors. The infection degree and duration from mild to severe and from acute to chronic determine the existence and forms of obtained lesions; cyst formation in the distorted epiphysis center is noticed only in severe cases while leukocytic cells infiltration is noticed in all forms. Associated microscopical lesions with different isolated microbes were demonstrated in detail in lesions score (Table 7).

\section{CONCLUSION}

Multiplex real-time PCR holds the potential to be a more reliable, economical, and rapid gold diagnostic tool for simultaneous detection of arthritis pathogens in turkey and provides an efficient way to ask several related epidemiological questions and also provides explanations for grossly and pathologically observed lesions accompanied with hematological and biochemical investigations.

\section{Conflict of interest}

The authors declare that they have no competing interests.

\section{Research ethics committee permission}

The current research work is permitted to be executed according to standards of Animal Research Ethics committee, Animal Health Research Institute (AHRI), Dokki, Egypt 


\section{Authors' contribution}

Azza S. El-Demerdash and Rehab E. Mowafy participated in the conception and design of the study, acquisition of data, and writing the paper and revising it critically for important intellectual contents. Rehab E. Mowafy and Sanaa M.Salem contributed in collection of samples and histopathological part. Sahar N.Mohamady contributed in measuring hematological and biochemical parameters. Azza S. El-Demerdash and Samah F.Ali performed microbiology part . Azza S. El-Demerdash and El-Toukhy E.I contributed in molecular part. All the authors have approved the final article version to be submitted.

\section{REFERENCES}

[1] Marcon AV, De Oliveira GF, Caldara FR, Garcia RG, Matins RA, Marcon A, et al. Bacteriological and Histopathological Evaluation of Articulations of Chickens Diagnosed with Arthritis. Brazilian J Poult Sci 2019;21. https://doi.org/10.1590/1806-9061-2018-0805

[2] Voleti PB, Buckley MR, Soslowsky L. Tendon healing: repair and regeneration. Annu Rev Biomed Eng 2012;14:47-71. https://doi.org/10.1146/annurev-bioeng-071811-150122

[3] Reck C, Menin Á, Canever MF, Pilati C, Miletti LC. Molecular detection of Mycoplasma synoviae and avian reovirus infection in arthritis and tenosynovitis lesions of broiler and breeder chickens in Santa Catarina State, Brazil. J S Afr Vet Assoc 2019;90. https://doi.org/10.4102/jsava.v90i0.1970

[4] Hassan AH, Hussein SA, AbdulAhad EA. Pathological and bacteriological study of bumblefoot cases in Sulaimaniyah province. Al-Anbar J Vet Sci 2012;5:195-201.

[5] Degernes LA, Lynch PS, Shivaprasad HL. Degenerative joint disease in captive waterfowl. Avian Pathol 2011;40:103-10. https://doi.org/10.1080/03079457.2010.541421

[6] Manley PN, Ancsin JB, Kisilevsky R. Rapid recycling of cholesterol: the joint biologic role of C-reactive protein and serum amyloid $A$. Med Hypotheses 2006;66:784-92. https://doi.org/10.1016/j.mehy.2005.10.018

[7] Pepys MB, Hirschfield GM. C-reactive protein: a critical update. J Clin Invest 2003;111:1805-12. https://doi.org/10.1172/JCI200318921

[8] Volanakis JE. Human C-reactive protein: expression, structure, and function. Mol Immunol 2001;38:189-97. https://doi.org/10.1016/\$0161-5890(01)00042-6

[9] Quinn PJ, Markey BK, Leonard FC, Hartigan P, Fanning S, Fitzpatrick Es. Veterinary microbiology and microbial disease. John Wiley \& Sons; 2011.

[10] CLSI (Clinical and Laboratory Standards Institute). M100-S23 Performance Standards for Antimicrobial. 2013.

[11] Thrall MA, Weiser G, Allison R, Campbell T. Veterinary hematology and clinical chemistry. John Wiley \& Sons; 2012.

[12] Banerjee M, Tripathi LM, Srivastava VML, Puri A, Shukla R. Modulation of inflammatory mediators by ibuprofen and curcumin treatment during chronic inflammation in rat. Immunopharmacol Immunotoxicol 2003;25:213-24. https://doi.org/10.1081/IPH-120020471

[13] Murray RL. Alanine aminotransferase. Clin Chem Theory, Anal Correl Kaplan LA, Pesce AJ (Eds), CV Mosby St Louis 1984;1090.

[14] Young DS. Effects of disease on Clinical Lab. Tests, 4th Ed AACC 2001.

[15] Burtis CA, Ashwood ER. Tietz textbook of clinical chemistry. Philadelphia 1999;1999:1654-5.

[16] Doumas BT, Bayse DD, Carter RJ, Peters T, Schaffer R. A candidate reference method for determination of total protein in serum. I. Development and validation. Clin Chem 1981;27:1642-50. https://doi.org/10.1093/clinchem/27.10.1642

[17] Drupt F. Colorimetric method for determination of albumin. Pharm Biol 1974;9:777-9.

[18] Suvarna K.S.; Layton, C.H.; Banchroft JD. Theory and practice of histological technique. 4th ed.; New York .Churchill; Livingston. J Virol
2013:8884-92.

[19] Manohar GR, Omprakash A V, Kanagaraju P. Leg weakness in commercial broiler chicken an overview. Int J Sci Environ Technol 2015;4:482-7.

[20] Kierończyk B, Rawski M, Józefiak D, Świlkatkiewicz S. Infectious and non-infectious factors associated with leg disorders in poultry--a review. Ann Anim Sci 2017;17:645-69. https://doi.org/10.1515/aoas2016-0098

[21] Amer MM, Mekky HM, Fedawy HS. Molecular identification of Mycoplasma synoviae from breeder chicken flock showing arthritis in Egypt. Vet World 2019;12:535. https://doi.org/10.14202/vetworld.2019.535-541

[22] Youssef FM, Soliman AA, Ibrahim GA, Saleh HA. Advanced Bacteriological Studies on Bumblefoot Infections in Broiler Chicken with Some Clinicopathological Alteration. Vetry Sci Rech 2019;1:1-9.

[23] Sellers HS. Current limitations in control of viral arthritis and tenosynovitis caused by avian reoviruses in commercial poultry. Vet Microbiol

2017:206:152-6. https://doi.org/10.1016/j.vetmic.2016.12.014

[24] Tawfik RG, Khalil SA, Ellakany HF, Torky HA. Mycoplasma Synoviae and other associated bacteria causing arthritis in chicken. Alexandria J Vet Sci 2016;49:163-9. https://doi.org/10.5455/ajvs.205876

[25] Agyare C, Boamah VE, Zumbi CN, Osei FB. Antibiotic use in poultry production and its effects on bacterial resistance. Antimicrob. Resist. Glob. Threat, IntechOpen; 2018 https://doi.org/10.5772/intechopen.79371

[26] Ghaderi R, Moradi Bidhendi S, Khaki P. Occurrence of multidrugresistant Salmonella enterica serovar Enteritidis isolates from poultry in Iran. Arch Razi Inst 2016;71:43-9.

[27] Kurutepe S, Surucuoglu S, Sezgin C, Gazi H, Gulay M, Ozbakkaloglu B. Increasing antimicrobial resistance in Escherichia coli isolates from community-acquired urinary tract infections during 1998-2003 in Manisa, Turkey. Jpn J Infect Dis 2005;58:159.

[28] Rahman H. Prevalence \& phenotypic expression of sopB gene among clinical isolates of Salmonella enterica. Indian J Med Res 2006;123:83.

[29] Günzel D, Kucharski LM, Kehres DG, Romero MF, Maguire ME. The MgtC virulence factor of Salmonella enterica serovar Typhimurium activates Na+, K+-ATPase. J Bacteriol 2006;188:5586-94. https://doi.org/10.1128/JB.00296-06

[30] Bugarel M, Granier SA, Weill F-X, Fach P, Brisabois A. A multiplex realtime PCR assay targeting virulence and resistance genes in Salmonella enterica serotype Typhimurium. BMC Microbiol 2011;11:151. https://doi.org/10.1186/1471-2180-11-151

[31] Lee S, Kim S, Lee H, Ha J, Lee J, Choi Y, et al. icaA Gene of Staphylococcus aureus Responds to $\mathrm{NaCl}$, Leading to Increased Biofilm Formation. J Food Prot 2018;81:412-6. https://doi.org/10.4315/0362-028X.JFP-17238

[32] Wang Y. The function of OmpA in Escherichia coli. Biochem Biophys Res Commun 2002;292:396-401. https://doi.org/10.1006/bbrc.2002.6657

[33] Sabharwal N, Dhall S, Chhibber S, Harjai K. Molecular detection of virulence genes as markers in Pseudomonas aeruginosa isolated from urinary tract infections. Int J Mol Epidemiol Genet 2014;5:125.

[34] Nahavandinejad M, Asadpour L, others. Mucoviscosity determination and detection of magA and rmpA genes in clinical isolates of Klebsiella pneumoniae in Northern Iran. Crescent J Med Biol Sci 2017;4:104-7.

[35] Campbell T. Exotic animal hematology and cytology. John Wiley \& Sons; 2015.

[36] Lucke M, Schmidmaier G, Sadoni S, Wildemann B, Schiller R, Stemberger $A$, et al. A new model of implant-related osteomyelitis in rats. J Biomed Mater Res Part B Appl Biomater An Off J Soc Biomater Japanese Soc Biomater Aust Soc Biomater Korean Soc Biomater 2003;67:593-602. https://doi.org/10.1002/jbm.b.10051

[37] Sohail MU, ljaz A, Yousaf MS, Ashraf K, Zaneb H, Aleem M, et al. Alleviation of cyclic heat stress in broilers by dietary supplementation of mannan-oligosaccharide and Lactobacillus-based probiotic: Dynamics of cortisol, thyroid hormones, cholesterol, C-reactive protein, and humoral immunity. Poult Sci 2010;89:1934-8. 
https://doi.org/10.3382/ps.2010-00751

[38] Campbell T, Coles E. Avian clinical Pathology,'in" Veterinary Clinical pathology". By EH Coles Fourth Ed London, Pp295 1986.

[39] Coles EH. Veterinary Clinical Pathology. WB Saunders Company. Philadelphia and London 1986.

[40] Zaki MS, Fawzy O, Osfor MH. Effect of E-coli OH157 on Baladi Broiler Chicken and some Biochemical studies. LIFE Sci JOURNAL-ACTA ZHENGZHOU Univ OVERSEAS Ed 2012;9:91-4.

[41] Echols MS. Evaluating and treating the kidneys. Clin Avian Med 2006;2:451-92.

[42] Chen F, Noll SL, Clanton CJ, Janni KA, Halvorson DA. Market turkey performance affected by floor type and brooding method. Appl Eng Agric 1991;7:606-12. https://doi.org/10.13031/2013.26277

[43] Davis JF, Kulkarni A, Fletcher O. Reovirus infections in young broiler chickens. Avian Dis 2013;57:321-5. https://doi.org/10.1637/10515021313-Case.1

[44] SharafEldin, Ibrahim TA. Turkey arthritis reovirus: pathogenesis and immune response 2015.

[45] Jones RC. Avian reovirus infections. Rev Sci Tech Int Des Epizoot 2000;19:614-9. https://doi.org/10.20506/rst.19.2.1237

[46] Huehn S, La Ragione RM, Anjum M, Saunders M, Woodward MJ, Bunge $\mathrm{C}$, et al. Virulotyping and antimicrobial resistance typing of Salmonella enterica serovars relevant to human health in Europe. Foodborne Pathog Dis 2010;7:523-35. https://doi.org/10.1089/fpd.2009.0447

[47] Wada M, Lkhagvadorj E, Bian L, Wang C, Chiba Y, Nagata S, et al. Quantitative reverse transcription-PCR assay for the rapid detection of methicillin-resistant Staphylococcus aureus. J Appl Microbiol 2010;108:779-88. https://doi.org/10.1111/j.1365-2672.2009.04476.x

[48] Ciftci A, Findik A, Onuk EE, Savasan S. Detection of methicillin resistance and slime factor production of Staphylococcus aureus in bovine mastitis. Brazilian J Microbiol 2009;40:254-61. https://doi.org/10.1590/S151783822009000200009

[49] Ewers C, Li G, Wilking H, Kie\$ $\beta$ \$ling S, Alt K, Antáo E-M, et al. Avian pathogenic, uropathogenic, and newborn meningitis-causing Escherichia coli: how closely related are they? Int J Med Microbiol 2007;297:163-76. https://doi.org/10.1016/j.ijmm.2007.01.003

[50] Compain F, Babosan A, Brisse S, Genel N, Audo J, Ailloud F, et al. Multiplex PCR for detection of seven virulence factors and K1/K2 capsular serotypes of Klebsiella pneumoniae. J Clin Microbiol 2014;52:4377-80. https://doi.org/10.1128/JCM.02316-14 